\title{
Electron Gas Dynamic Conductivity Tensor on the Nanotube Surface in Magnetic Field
}

\author{
A. M. Ermolaev, S. V. Kofanov, and G. I. Rashba \\ Theoretical Physics Department, V.N. Karazin Kharkiv National University, 4 Svobody square, 61077 Kharkiv, Ukraine
}

Correspondence should be addressed to G. I. Rashba, georgiy.i.rashba@univer.kharkov.ua

Received 14 June 2010; Revised 17 January 2011; Accepted 11 February 2011

Academic Editor: Sergio E. Ulloa

Copyright (๑) 2011 A. M. Ermolaev et al. This is an open access article distributed under the Creative Commons Attribution License, which permits unrestricted use, distribution, and reproduction in any medium, provided the original work is properly cited.

Kubo formula was derived for the electron gas conductivity tensor on the nanotube surface in longitudinal magnetic field considering spatial and time dispersion. Components of the degenerate and nondegenerate electron gas conductivity tensor were calculated. The study has showed that under high electron density, the conductivity undergoes oscillations of de Haas-van Alphen and Aharonov-Bohm types with the density of electrons and magnetic field changes.

\section{Introduction}

Electromagnetic waves in the systems with cylindrical geometry have been studied for a long time [1-3]. The authors of the work [4] had solved the Maxwell's equation taking into account the retardation for the conducting cylinder dipped in a dielectric medium. They considered time dispersion of the dielectric permittivity of conductor and dielectric. Also they had solved the dispersion equation for the wave spectrum and obtained new branches in the spectrum of surface polaritons. The paper [5] deals with polaritons in the magnetic wire with uniaxial anisotropy, dipped into a dielectric medium. Wire's dielectric and magnetic permittivity tensor frequency dependence also was studied. Polaritons spectra were found. Constant, parallel to the magnetic wire axis, external magnetic field effect was studied in paper [6]. In this paper, how wave characteristics allow one to obtain data about the material structure was shown.

A necessary of semiconductor nanotubes waveguide characteristics researching is caused by growing interest [710] to these systems. Plasma and magnetoplasma waves propagation on the nanotube surface were studied in [1118]. They focused on cylindrical geometry systems calculations. These calculations were made by means of matter dielectric permittivity simple models. The problem is that authors of [11-18] have not used dielectric permittivity and conductivity tensors exact expressions. Also they have not taken into account the time and spatial dispersion. However, these exact formulas may significantly influence the tube waveguide characteristics expressions.

In this paper, we present conductivity tensor components calculation and their wave vector and frequency dependencies of the following system: electron gas on the nanotube surface, affected by a parallel to the tube axis external magnetic field. In Section 2, Kubo formula was obtained for the conductivity tensor. In Sections 3 and 4 we considered degenerate electron gas, and in Section 5, nondegenerate gas.

\section{Dynamic Conductivity Tensor}

Confined by the nanotube's cylindrical surface, electron gas imbedded into a parallel to the cylinder axis magnetic field has axial symmetry. Therefore, it is suitable to characterize the electron state by the conserved quantities: angular momentum projection $m=0, \pm 1, \ldots$, impulse projection $k$ of electron on tube axis $z$, and spin quantum number $\sigma= \pm 1$. Electron stationary state $|m k \sigma\rangle$ 
wave function and its energy spectrum are equal [11-14] to

$$
\begin{gathered}
\Psi_{m k \sigma}(\varphi, z, \alpha)=\frac{1}{\sqrt{S}} e^{i(m \varphi+k z)} \chi_{\sigma}(\alpha), \\
\varepsilon_{m k \sigma}=\varepsilon_{0}\left(m+\frac{\Phi}{\Phi_{0}}\right)^{2}+\frac{k^{2}}{2 m_{*}}+\sigma \mu_{B} B,
\end{gathered}
$$

where $m_{*}$ and $\mu_{B}$ are the effective mass and the electron spin magnetic moment, $\varphi$ and $z$ are cylindrical coordinates, $\chi_{\sigma}(\alpha)$ is a spin wave function, $\varepsilon_{0}=1 / 2 m_{*} a^{2}$ is a rotational quantum, $a$ is the tube radius, $\Phi=\pi a^{2} B$ is the magnetic induction $B$ flux through the tube section, $\Phi_{0}=2 \pi c / e$ is flux quantum, $S=2 \pi a L$ is the nanotube lateral surface area, and the length of nanotube is $L$. Hereinafter, the quantum constant is considered to equal unity. The spectrum (2) is a set of one-dimensional subzones, whose boundaries $\mathcal{E}_{m 0 \sigma}$ are not equidistant.

One can expand the current electron density operator in field $B$ into series of cylindrical harmonics (1):

$$
\mathbf{J}(\varphi, z)=\sum_{m k} \mathbf{J}(m, k) \Psi_{m k}(\varphi, z)
$$

where

$$
\mathbf{J}(m, k)=e \sum_{m_{1} k_{1} m_{2} k_{2} \sigma}\left\langle m_{1} k_{1}|\mathbf{V}(-m,-k)| m_{2} k_{2}\right\rangle a_{m_{1} k_{1} \sigma}^{+} a_{m_{2} k_{2} \sigma} .
$$

Here $a_{m k \sigma}$ and $a_{m k \sigma}^{+}$are annihilation and creation operators of electrons in state $|m k \sigma\rangle$, and $\left\langle m_{1} k_{1}|\mathbf{V}(-m,-k)| m_{2} k_{2}\right\rangle$ are operator $\mathbf{V}$ matrix elements

$$
\begin{aligned}
\mathbf{V}(-m,-k)=\frac{1}{2 m_{*}}[ & \left(-i \nabla-\frac{e}{c} \mathbf{A}\right) \Psi_{m k}^{*}(\varphi, z) \\
& \left.+\Psi_{m k}^{*}(\varphi, z) \times\left(-i \nabla-\frac{e}{c} \mathbf{A}\right)\right]
\end{aligned}
$$

in the functional basis (1), $\mathbf{B}=\operatorname{rot} \mathbf{A}$. The operator's (4) cylindrical components are equal to

$$
\begin{gathered}
J_{\varphi}(m, k)=\frac{e}{m_{*} a \sqrt{S}} \sum_{m^{\prime} k^{\prime} \sigma}\left(m^{\prime}+\frac{\Phi}{\Phi_{0}}+\frac{m}{2}\right) a_{m^{\prime} k^{\prime} \sigma}^{+} a_{\left(m^{\prime}+m\right)\left(k^{\prime}+k\right) \sigma} \\
J_{z}(m, k)=\frac{e}{m_{*} \sqrt{S}} \sum_{m^{\prime} k^{\prime} \sigma}\left(k^{\prime}+\frac{k}{2}\right) a_{m^{\prime} k^{\prime} \sigma}^{+} a_{\left(m^{\prime}+m\right)\left(k^{\prime}+k\right) \sigma} .
\end{gathered}
$$

Within the framework of electron gas linear response theory $[19,20]$ to the weak electric field $\mathbf{E}$, changes with wave vector $q$ and frequency $\omega$, it is possible to obtain the current density

$$
j_{\mu}(m, q, \omega)=\sum_{\nu} \sigma_{\mu \nu}(m, q, \omega) E_{\nu}(m, q, \omega)
$$

where

$$
\begin{aligned}
\sigma_{\mu \nu}(m, q, \omega)= & i \frac{e^{2} n}{m_{*} \omega} \delta_{\mu \nu}+\frac{1}{\omega} \int_{0}^{\infty} d t e^{i \omega t} \\
& \times\left\langle\left[J_{\mu}(m, q, t), J_{\nu}(-m,-q, 0)\right]\right\rangle,
\end{aligned}
$$

Kubo formula for conductivity tensor. Here $J_{\mu}(m, q, t)$ is a component of Heisenberg operator (6), $n$ is a surface electron density, $[a, b]=a b-b a$, and angle brackets mark Gibbs average.

We have substituted (6) in (8) and obtained the relation between the conductivity tensor and Fourier time component of two-electron Greens function $[19,20]$ :

$$
K_{t}(1,2 ; 3,4)=-i \theta(t)\left\langle\left[a_{1}^{+}(t) a_{2}(t), a_{3}^{+}(0) a_{4}(0)\right]\right\rangle
$$

where $1=\left(m_{1}, k_{1}, \sigma_{1}\right), \ldots, \theta(t)$ is a Heaviside's function. In case of free electron gas, function (9) is expressed by one-electron Green function. In the result, the conductivity tensor components (8) are equal to

$$
\begin{aligned}
& \sigma_{\varphi \varphi}(m, q, \omega) \\
& =i \frac{e^{2} n}{m_{*} \omega}+\frac{i e^{2}}{m_{*}^{2} a^{2} \omega S} \\
& \cdot \sum_{m^{\prime} k \sigma} f\left(\varepsilon_{m^{\prime} k \sigma}\right)\left[\frac{\left(m^{\prime}+\Phi / \Phi_{0}+m / 2\right)^{2}}{\varepsilon_{m^{\prime} k \sigma}-\varepsilon_{\left(m^{\prime}+m\right)(k+q) \sigma}+\omega+i 0}\right. \\
& \left.-\frac{\left(m^{\prime}+\Phi / \Phi_{0}-m / 2\right)^{2}}{\varepsilon_{\left(m^{\prime}-m\right)(k-q) \sigma}-\varepsilon_{m^{\prime} k \sigma}+\omega+i 0}\right], \\
& \sigma_{\varphi z}(m, q, \omega) \\
& =\sigma_{\varphi z}(m, q, \omega) \\
& =\frac{i e^{2}}{m_{*}^{2} a \omega S} \sum_{m^{\prime} k \sigma} f\left(\varepsilon_{m^{\prime} k \sigma}\right) \\
& \cdot\left[\frac{\left(m^{\prime}+\Phi / \Phi_{0}+m / 2\right)(k+q / 2)}{\varepsilon_{m^{\prime} k \sigma}-\varepsilon_{\left(m^{\prime}+m\right)(k+q) \sigma}+\omega+i 0}\right. \\
& \left.-\frac{\left(m^{\prime}+\Phi / \Phi_{0}-m / 2\right)(k-q / 2)}{\varepsilon_{\left(m^{\prime}-m\right)(k-q) \sigma}-\varepsilon_{m^{\prime} k \sigma}+\omega+i 0}\right], \\
& \sigma_{z z}(m, q, \omega) \\
& =i \frac{e^{2} n}{m_{*} \omega}+\frac{i e^{2}}{m_{*}^{2} \omega S} \sum_{m^{\prime} k \sigma} f\left(\varepsilon_{m^{\prime} k \sigma}\right) \\
& {\left[\frac{(k+q / 2)^{2}}{\varepsilon_{m^{\prime} k \sigma}-\varepsilon_{\left(m^{\prime}+m\right)(k+q) \sigma}+\omega+i 0}\right.} \\
& \left.-\frac{(k-q / 2)^{2}}{\varepsilon_{\left(m^{\prime}-m\right)(k-q) \sigma}-\varepsilon_{m^{\prime} k \sigma}+\omega+i 0}\right],
\end{aligned}
$$

where $f(\varepsilon)$ is Fermi function. These components are included into the dispersion equation for electromagnetic waves, propagating along the tube.

In case of $a \rightarrow \infty$, one can obtain from (10) the wellknown Drude-Lorentz expression, which describes twodimensional electron gas with the magnetic field absence: $\sigma_{\varphi \varphi}=\sigma_{z z}=i e^{2} n / m_{*} \omega, \sigma_{\varphi z}=0$. The real part of conductivity tensor in this case is equal to zero. It is caused by not taking into account the electrons collisions. That effect can 
be considered by means of $\omega \rightarrow \omega+i v_{c}$ replacement in (10). Here $v_{c}$ is the collisions frequency.

\section{Degenerate Gas}

At zero temperature, integrals over $k$, included into (10), can be calculated exactly. Thus, real and imaginary parts of conductivity (10) are equal to

$$
\begin{aligned}
\operatorname{Re} \sigma_{\varphi \varphi}(m, q, \omega)=- & \frac{e^{2}}{4 \pi m_{*}|q| a^{3} \omega} \\
& \times \sum_{m^{\prime} \sigma}\left\{\left(m^{\prime}+\frac{\Phi}{\Phi_{0}}+\frac{m}{2}\right)^{2}\right. \\
& \times\left[\theta\left(A_{+-+}\right)-\theta\left(A_{--+}\right)\right] \\
& -\left(m^{\prime}+\frac{\Phi}{\Phi_{0}}-\frac{m}{2}\right)^{2} \\
\operatorname{Re} \sigma_{\varphi z}(m, q, \omega)=-\frac{e^{2}}{4 \pi(}(q a)^{2} \omega & \\
& \left.\times \sum_{m^{\prime} \sigma}\left\{\left(m_{++-}^{\prime}+\frac{\Phi}{\Phi_{0}}+\frac{m}{2}\right)\left(\omega-A_{-+-}\right)\right]\right\} \\
& \times\left[\theta\left(A_{+-+}\right)-\theta\left(A_{--+}\right)\right] \\
& -\left(m^{\prime}+\frac{\Phi}{\Phi_{0}}-\frac{m}{2}\right)\left(\omega-\Omega_{-}\right) \\
&
\end{aligned}
$$

$$
\begin{aligned}
\operatorname{Re} \sigma_{z z}(m, q, \omega)= & -\frac{m_{*} e^{2}}{4 \pi|q|^{3} a \omega} \\
& \times \sum_{m^{\prime} \sigma}\left\{\left(\omega-\Omega_{+}\right)^{2}\left[\theta\left(A_{+-+}\right)-\theta\left(A_{--+}\right)\right]\right. \\
& \left.\quad-\left(\omega-\Omega_{-}\right)^{2}\left[\theta\left(A_{++-}\right)-\theta\left(A_{-+-}\right)\right]\right\},
\end{aligned}
$$

$$
\begin{aligned}
\operatorname{Im} \sigma_{\varphi \varphi}(m, q, \omega)= & \frac{e^{2} n}{m_{*} \omega}-\frac{e^{2}}{4 \pi^{2} m_{*} q a^{3} \omega} \\
& \times \sum_{m^{\prime} \sigma}\left[\left(m^{\prime}+\frac{\Phi}{\Phi_{0}}+\frac{m}{2}\right)^{2} \ln \left|\frac{A_{+-+}}{A_{--+}}\right|\right. \\
& \left.\quad-\left(m^{\prime}+\frac{\Phi}{\Phi_{0}}-\frac{m}{2}\right)^{2} \ln \left|\frac{A_{++-}}{A_{-+-}}\right|\right],
\end{aligned}
$$

$$
\begin{aligned}
\operatorname{Im} \sigma_{\varphi z}(m, q, \omega)= & -\frac{e^{2} m n}{m_{*} q a \omega}-\frac{e^{2}}{4 \pi^{2}(q a)^{2} \omega} \\
& \times \sum_{m^{\prime} \sigma}\left[\left(m^{\prime}+\frac{\Phi}{\Phi_{0}}+\frac{m}{2}\right)\left(\omega-\Omega_{+}\right)\right. \\
& \times \ln \left|\frac{A_{+-+}}{A_{--+}}\right|-\left(m^{\prime}+\frac{\Phi}{\Phi_{0}}-\frac{m}{2}\right) \\
\operatorname{Im} \sigma_{z z}(m, q, \omega)= & \left.-\frac{2 e^{2} \varepsilon_{0} m^{2} n}{q^{2} \omega}-\frac{m_{*} e^{2}}{4 \pi^{2} q^{3} a \omega}\left|\frac{A_{++-}}{A_{-+-}}\right|\right], \\
& \times \sum_{m^{\prime} \sigma}\left[\left(\omega-\Omega_{+}\right)^{2} \ln \left|\frac{A_{+-+}}{A_{--+}}\right|\right. \\
& \left.\quad-\left(\omega-\Omega_{-}\right)^{2} \ln \left|\frac{A_{++-}}{A_{-+-}}\right|\right],
\end{aligned}
$$

where

$$
\begin{gathered}
A_{+-+}=q v_{m^{\prime}}^{\sigma}-\omega_{-}+\Omega_{+}, \quad A_{--+}=-q v_{m^{\prime}}^{\sigma}-\omega_{-}+\Omega_{+}, \\
A_{++-}=q v_{m^{\prime}}^{\sigma}-\omega_{+}+\Omega_{-}, \quad A_{-+-}=-q v_{m^{\prime}}^{\sigma}-\omega_{+}+\Omega_{-}, \\
v_{m^{\prime}}^{\sigma}=\sqrt{\frac{2}{m_{*}}}\left(\varepsilon_{F}-\varepsilon_{m^{\prime} \sigma}\right)^{1 / 2}
\end{gathered}
$$

is the electron motion along the tube axis velocity maximum in subzone $\left(m^{\prime}, \sigma\right), \varepsilon_{m^{\prime} \sigma}=\varepsilon_{m^{\prime} 0 \sigma}$ is the subzone boundary, $\varepsilon_{F}$ is the Fermi energy, $\omega_{ \pm}=\omega \pm q^{2} / 2 m_{*}$, and

$$
\Omega_{ \pm}\left(m, m^{\prime}\right)=\varepsilon_{0}\left[ \pm\left(m^{\prime}+\frac{\Phi}{\Phi_{0}} \pm m\right)^{2} \mp\left(m^{\prime}+\frac{\Phi}{\Phi_{0}}\right)^{2}\right]
$$

is the frequency of the "vertical" electron transitions between the spectrum (2) subzones. While obtaining (12) out of (10), the identity below was used

$$
\frac{B^{2}}{B-C}=B+C+\frac{C^{2}}{B-C} \text {. }
$$

\section{Longitudinal Conductivity}

This section concentrates on the analysis of degenerate electron gas longitudinal conductivity, (11) (12), assuming $m=0$. The numerical calculations were carried out also for $m=1$ case. In this case, electrons make only intra-subzone transitions without spin change. Longitudinal conductivity is included into the propagating along the nanotube axis intrasubzone magnetoplasma waves dispersion equation [13], because it is related to the polarization operator $P$ by the relation below

$$
\operatorname{Im} \sigma_{z z}(0, q, \omega)=\frac{e^{2} \omega}{q^{2}} \operatorname{Re} P(0, q, \omega)
$$


In case of $m=0$, we obtain the following equations from (11) and (12):

$$
\begin{aligned}
& \operatorname{Re} \sigma_{z z}(q, \omega)=-\frac{e^{2} m_{*} \omega}{4 \pi|q|^{3} a} \\
& \times \sum_{m^{\prime} \sigma}\left[\theta\left(q v_{m^{\prime}}^{\sigma}-\omega_{-}\right)-\theta\left(-q v_{m^{\prime}}^{\sigma}-\omega_{-}\right)\right. \\
&\left.-\theta\left(q v_{m^{\prime}}^{\sigma}-\omega_{+}\right)+\theta\left(-q v_{m^{\prime}}^{\sigma}-\omega_{+}\right)\right], \\
& \operatorname{Im} \sigma_{z z}(q, \omega)=-\frac{e^{2} m_{*} \omega}{4 \pi^{2} q^{3} a} \sum_{m^{\prime} \sigma}\left(\ln \left|\frac{q v_{m^{\prime}}^{\sigma}-\omega_{-}}{-q v_{m^{\prime}}^{\sigma}-\omega_{-}}\right|\right. \\
&\left.\quad-\ln \left|\frac{q v_{m^{\prime}}^{\sigma}-\omega_{+}}{-q v_{m^{\prime}}^{\sigma}-\omega_{+}}\right|\right) .
\end{aligned}
$$

Suppose that the flux ratio in (2) should be equal to $\Phi / \Phi_{0}=$ $M+\eta$, where $M=0,1, \ldots$ is an integer part of $\Phi / \Phi_{0}$ and $\eta$ is a fractional part $(0 \leq \eta<1)$. One can use the achievable in experiments values of carbon and semiconductor nanotube radii and obtain that the value of $M$ in the accessible magnetic fields is low, unless the tube radius is too large. Then the energy of electron (2) is minimal in the subzone $\left(m^{\prime}=-M, \sigma=-1\right)$ :

$$
\varepsilon_{\min }=\varepsilon_{0} \eta^{2}-\mu_{B} B
$$

In this case, the electron rotation energy is nearly compensated by the rotation in the magnetic field. Usually spin level splitting (2) is small, that is,

$$
2 \mu_{B} B<\varepsilon_{0}(1+2 \eta),
$$

and the electron density corresponds to inequality

$$
n<\frac{\sqrt{m_{*} \mu_{B} B}}{\pi^{2} a} .
$$

Then electrons partially fill only the lower subzone $(-M,-1)$. Therefore, (17) leads to the following:

$$
\begin{gathered}
\operatorname{Re} \delta \sigma=-\frac{\pi}{2} \frac{k_{0}}{q} x^{2}, \\
\operatorname{Im} \delta \sigma=-\frac{k_{0} x^{2}}{2 q} \ln \left|\frac{\left(1+q / 2 k_{0}\right)^{2}-x^{2}}{\left(1-q / 2 k_{0}\right)^{2}-x^{2}}\right|,
\end{gathered}
$$

where

$$
\begin{gathered}
\delta \sigma=\sigma_{z z}(q, \omega) \frac{m_{*} \omega}{e^{2} n}, \quad x=\frac{\omega}{q v_{0}}, \\
v_{0}=\frac{k_{0}}{m_{*}}=\sqrt{\frac{2}{m_{*}}}\left(\varepsilon_{F}+\mu_{B} B-\varepsilon_{0} \eta^{2}\right)^{1 / 2}
\end{gathered}
$$

is the electrons velocity maximum in subzone $(-M,-1)$. Figures 1 and 2 show the $x=\omega / q v_{0}$ dependencies of $\operatorname{Re} \delta \sigma$ and $\operatorname{Im} \delta \sigma$ under $\eta=0.01, q=k_{0}$, and $k_{0} a=1$ in quantum limit, when Fermi energy is located in subzone $(-M,-1)$.

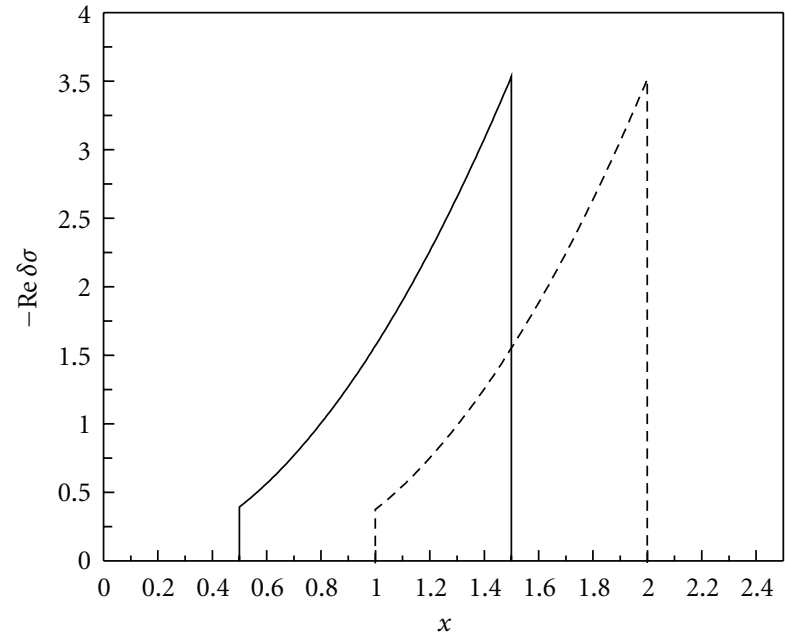

$$
\begin{array}{r}
m=0 \\
--m=1
\end{array}
$$

Figure 1: Dependence of $\operatorname{Re} \delta \sigma$ on $x=\omega / q v_{0}$ under $\eta=0.01$, $q=k_{0}, k_{0} a=1, m=0$ (solid curve), and $m=1$ (dashed).

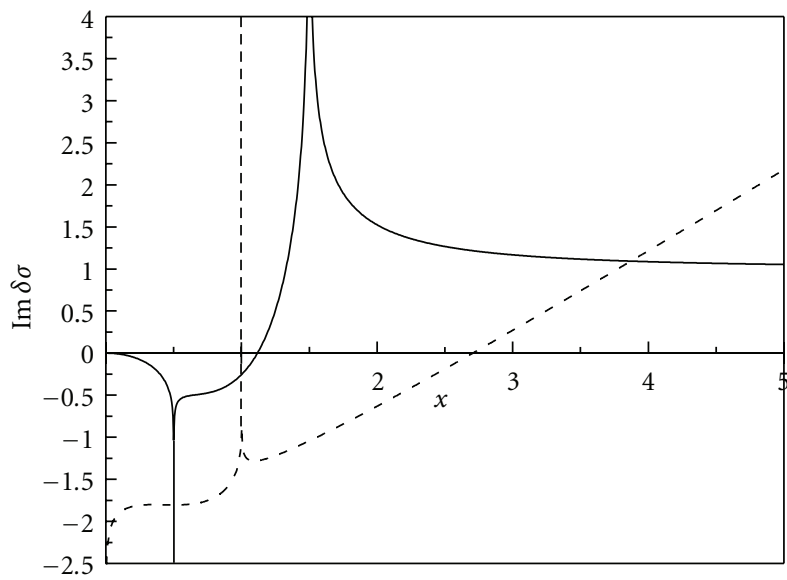

$$
\begin{aligned}
-m & =0 \\
--m & =1
\end{aligned}
$$

Figure 2: Dependence of $\operatorname{Im} \delta \sigma$ on $x=\omega / q v_{0}$ under $\eta=0.01$, $q=k_{0}, k_{0} a=1, m=0$ (solid curve), and $m=1$ (dashed).

One can calculate what included into (17) sum by $m^{\prime}$ using a Poisson formula, assuming $\varepsilon_{F} \gg \varepsilon_{0}$, that is, a large quantity of subzones are filled. Within long wavelength assumption $q v_{F} \ll \omega\left(v_{F}\right.$ is a Fermi velocity), we obtain the following formula from (17):

$$
\begin{aligned}
\operatorname{Im} \sigma_{z z}=\frac{e^{2} n}{m_{*} \omega}[ & +\frac{2}{\pi^{2}}\left(\frac{\varepsilon_{0}}{\varepsilon_{F}}\right)^{3 / 4} \sum_{l=1}^{\infty} \frac{1}{l 3 / 2} \\
& \left.\times \sin \left(2 \pi l \sqrt{\frac{\varepsilon_{F}}{\varepsilon_{0}}}-\frac{\pi}{4}\right) \cos \left(2 \pi l \frac{\Phi}{\Phi_{0}}\right)\right] .
\end{aligned}
$$


Here we neglected the spin level splitting (2). Monotonous part of conductivity (23) under the given electron density does not depend on the tube radius and coincides with the two-dimensional electron gas conductivity [21]. It is within the $a \rightarrow \infty$, when the tube is cut along its generatrix and unrolled onto a plane. Conductivity (23) experiences the Aharonov-Bohm and de Haas-van Alphen oscillation types. The reason of the de Haas-van Alphen oscillation types existance is that the electron states density has root peculiarities on the subzone bounaries. Change of the tube radius or electron density causes these peculiarities to pass throw Fermi energy and de Haas-van Alphen oscillation types to appear. If we study conductivity dependence (23) on $\varepsilon_{F}^{1 / 2}=(n / v)^{1 / 2}$ ( $v$ is the two-dimensional electron gas states density), then the oscillation period is $1 / \sqrt{2 m_{*}} a$. If we study the radius dependence, the period of conductivity oscillations is equal to $1 / \sqrt{2 m_{*} \varepsilon_{F}}$. Aharonov-Bohm oscillations are caused by the magnetic flux through the tube crosssection change. Their period is equal to the flux quantum. Figure 3 shows the dependence of the (23) first conductivity harmonic on $\left(\varepsilon_{F} / \varepsilon_{0}\right)^{1 / 2}$ under $\Phi / \Phi_{0}=0.01$.

\section{Nondegenerate Electron Gas}

Nondegenerate electron gas emerges if inequality $\beta|\mu| \gg 1$ is true on the semiconductor nanotube surface. Here $\beta$ is a reverse temperature and $\mu$ is a chemical potential. Using Boltzmann's statistics, the chemical potential is related to the electron density as

$$
n=\frac{1}{\pi^{3 / 2} a} \sqrt{\frac{m_{*}}{2 \beta}} e^{\beta \mu} \operatorname{ch}\left(\beta \mu_{B} B\right) \sum_{m=-\infty}^{\infty} e^{-\beta \varepsilon_{m}}
$$

where $\varepsilon_{m}=\varepsilon_{0}\left(m+\Phi / \Phi_{0}\right)^{2}$. The included here summation may be transformed by means of formula [22]

$$
\sum_{m=-\infty}^{\infty} e^{-x(m+v)^{2}}=\sqrt{\frac{\pi}{x}} \sum_{l=-\infty}^{\infty} e^{-\pi^{2} l^{2} x} \cos (2 \pi l v), \quad x>0 .
$$

Real and imaginary tensor (10) parts in the studied case assuming $m=0$ are equal to

$$
\begin{aligned}
& \operatorname{Re} \sigma_{\varphi \varphi}(q, \omega) \\
& =\frac{e^{2}}{4 \sqrt{\pi} m_{*}\left(\beta \varepsilon_{0}\right)^{3 / 2}|q| a^{3} \omega} e^{\beta \mu} \operatorname{ch}\left(\beta \mu_{B} B\right) \\
& \quad \times\left\{\exp \left[-\frac{m_{*} \beta}{2}\left(\frac{\omega_{-}}{q}\right)^{2}\right]-\exp \left[-\frac{m_{*} \beta}{2}\left(\frac{\omega_{+}}{q}\right)^{2}\right]\right\} \\
& \quad \times \sum_{l=-\infty}^{\infty} \exp \left(-\frac{\pi^{2} l^{2}}{\beta \varepsilon_{0}}\right)\left(1-\frac{2 \pi^{2} l^{2}}{\beta \varepsilon_{0}}\right) \cos \left(2 \pi l \frac{\Phi}{\Phi_{0}}\right),
\end{aligned}
$$

$$
\begin{aligned}
& \operatorname{Re} \sigma_{\varphi z}(q, \omega) \\
& =\frac{\sqrt{\pi} e^{2}}{2\left(\beta \varepsilon_{0}\right)^{3 / 2}(q a)^{2}} e^{\beta \mu} \operatorname{ch}\left(\beta \mu_{B} B\right) \\
& \quad \times \sum_{l=-\infty}^{\infty} l \exp \left(-\frac{\pi^{2} l^{2}}{\beta \varepsilon_{0}}\right) \\
& \quad \times\left\{\exp \left[-\frac{m_{*} \beta}{2}\left(\frac{\omega_{-}}{q}\right)^{2}\right]-\exp \left[-\frac{m_{*} \beta}{2}\left(\frac{\omega_{+}}{q}\right)^{2}\right]\right\} \\
& \quad \times \sin \left(2 \pi l \frac{\Phi}{\Phi_{0}}\right)
\end{aligned}
$$

$\operatorname{Re} \sigma_{z z}(q, \omega)$$$
\begin{aligned}
= & \frac{e^{2} n \omega}{|q|^{3}} \sqrt{\frac{\pi m_{*} \beta}{2}} \\
& \times\left\{\exp \left[-\frac{m_{*} \beta}{2}\left(\frac{\omega_{-}}{q}\right)^{2}\right]-\exp \left[-\frac{m_{*} \beta}{2}\left(\frac{\omega_{+}}{q}\right)^{2}\right]\right\},
\end{aligned}
$$$$
\operatorname{Im} \sigma_{\varphi \varphi}(q, \omega)
$$$$
\begin{aligned}
= & \frac{e^{2} n}{m_{*} \omega}+\frac{e^{2}}{4 \pi m_{*}\left(\beta \varepsilon_{0}\right)^{3 / 2} q a^{3} \omega} e^{\beta \mu} \operatorname{ch}\left(\beta \mu_{B} B\right) \\
& \times\left[F\left(\sqrt{\frac{m_{*} \beta}{2}} \frac{\omega_{-}}{q}\right)-F\left(\sqrt{\frac{m_{*} \beta}{2}} \frac{\omega_{+}}{q}\right)\right] \\
& \times \sum_{l=-\infty}^{\infty} \exp \left(-\frac{\pi^{2} l^{2}}{\beta \varepsilon_{0}}\right)\left(1-\frac{2 \pi^{2} l^{2}}{\beta \varepsilon_{0}}\right) \cos \left(2 \pi l \frac{\Phi}{\Phi_{0}}\right),
\end{aligned}
$$$$
\operatorname{Im} \sigma_{\varphi z}(q, \omega)
$$$$
=\frac{e^{2}}{2\left(\beta \varepsilon_{0}\right)^{3 / 2}(a q)^{2}} e^{\beta \mu} \operatorname{ch}\left(\beta \mu_{B} B\right)
$$$$
\times \sum_{l=-\infty}^{\infty} l \exp \left(-\frac{\pi^{2} l^{2}}{\beta \varepsilon_{0}}\right)
$$$$
\times\left\{F\left(\sqrt{\frac{m_{*} \beta}{2}} \frac{\omega_{-}}{q}\right)-F\left(\sqrt{\frac{m_{*} \beta}{2}} \frac{\omega_{+}}{q}\right)\right\}
$$$$
\times \sin \left(2 \pi l \frac{\Phi}{\Phi_{0}}\right),
$$

$\operatorname{Im} \sigma_{z z}(q, \omega)$

$$
\begin{aligned}
= & \frac{e^{2} n \omega}{q^{3}} \sqrt{\frac{m_{*} \beta}{2}} \\
& \times\left[F\left(\sqrt{\frac{m_{*} \beta}{2}} \frac{\omega_{-}}{q}\right)-F\left(\sqrt{\frac{m_{*} \beta}{2}} \frac{\omega_{+}}{q}\right)\right],
\end{aligned}
$$




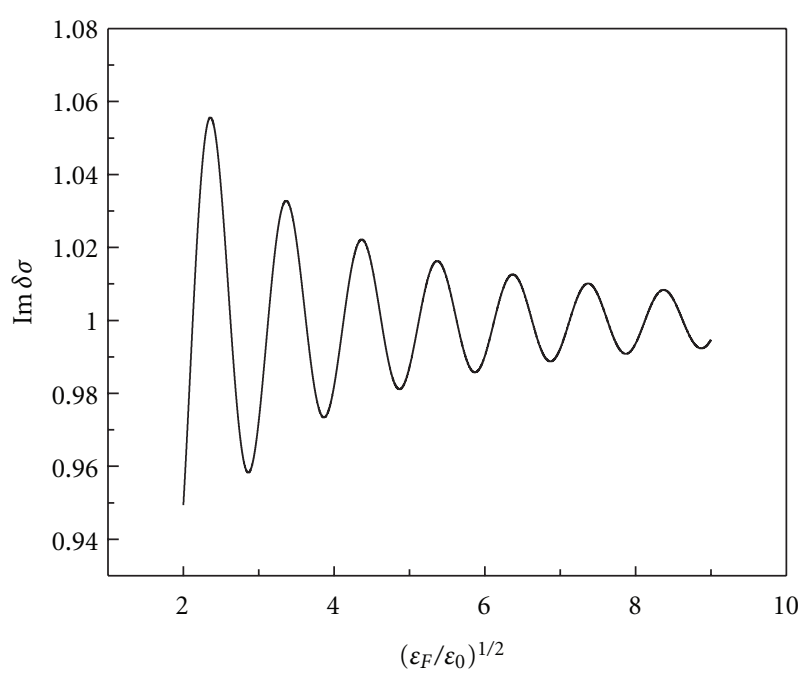

FIGURE 3: Dependence of the first harmonic $\operatorname{Im} \delta \sigma(23)$ on $\left(\varepsilon_{F} / \varepsilon_{0}\right)^{1 / 2}$ under $\Phi / \Phi_{0}=0.01$.

where

$$
F(x)=\frac{1}{\sqrt{\pi}} p \int_{-\infty}^{\infty} d y \frac{e^{-y^{2}}}{x-y}
$$

Components $\sigma_{\varphi \varphi}$ and $\sigma_{\varphi z}$ in (26) are expressed through $\beta$ and $\mu$, that is, they refer to an open system of tube surface electrons. They experience only Aharonov-Bohm oscillations with the magnetic field changes. AharonovBohm oscillations are not taking place in $\sigma_{z z}$ component while it is described in $\beta$ and $n$ terms.

\section{Conclusion}

Simplified conductivity models are usually used for studying electromagnetic waves propagation in the cylindrical geometry systems, for example, in nanotubes. The metal cylinder conductivity is often believed to be endless, and the dielectric permittivity of the matter, where cylinder is dipped, is considered to be constant or only frequency dependent. Conductivity's spatial dispersion as a rule is not taken into account. Nonetheless, the electromagnetic field's nature in the tube and its waveguide characteristics are sensitive to the surface currents. Therefore, the electron gas conductivity tensor components calculation problem, taking into account spatial and time dispersion, is worth consideration. It is given in this work conductivity tensor components may be used in obtaining a dispersion equation for electromagnetic waves in the tube. The example of longitudinal conductivity showed what data of electron gas is possible to obtain by measuring conductivity. Particularly, imaginary part of conductivity experiences de Haas-van Alphen and Aharonov-Bohm oscillations types with the electron density and magnetic field changes. The oscillation periods measurement enables one to identify the electron effective mass and the combination of universal constants included into flux quantum.

\section{Acknowledgment}

The authors would like to thank T. Rashba for help with preparation of the paper.

\section{References}

[1] J. A. Stratton, Electromagnetic Theory, McGraw-Hill, New York, NY, USA, 1941.

[2] J. D. Jackson, Classical Electrodynamics, John Wiley \& Sons, New York, NY, USA, 1962.

[3] L. D. Landau and E. M. Lifshitz, Electrodynamics of Continuous Media, Pergamon, Oxford, UK, 1971.

[4] C. A. Pfeiffer, E. N. Economou, and K. L. Ngai, "Surface polaritons in a circularly cylindrical interface: surface plasmons," Physical Review B, vol. 10, no. 8, pp. 3038-3051, 1974.

[5] E. F. Vasconcelos, N. T. Oliveira, G. A. Farias, and N. S. Almeida, "Polaritons confined in magnetic wires," Physical Review B, vol. 44, no. 22, pp. 12621-12623, 1991.

[6] N. S. Almeida, G. A. Farias, N. T. Oliveira, and E. F. Vasconcelos, "Influence of a dc field on polaritons confined in magnetic wires," Physical Review B, vol. 48, no. 13, pp. 98399842, 1993.

[7] S. Iijima, "Helical microtubules of graphitic carbon," Nature, vol. 354, no. 6348, pp. 56-58, 1991.

[8] M. S. Dresselhaus, G. Dresselhaus, and P. C. Eklund, Science of Fullerens and Carbon Nanotubes, Academic Press, New York, NY, USA, 1996.

[9] R. Saito, G. Dresselhaus, and M. S. Dresselhaus, Physical Properties of Carbon Nanotubes, Imperial College Press, London, UK, 1998.

[10] L. I. Magarill, A. V. Chaplik, and M. V. Entin, "Spectrum and kinetics of electrons in curved nanostructures," Uspekhi Fizicheskikh Nauk, vol. 175, no. 9, pp. 995-1000, 2005.

[11] M. F. Lin and K. W. K. Shung, "Elementary excitations in cylindrical tubules," Physical Review B, vol. 47, no. 11, pp. 6617-6624, 1993.

[12] O. Sato, Y. Tanaka, M. Kobayashi, and A. Hasegawa, "Exotic behavior of the dielectric function and the plasmons of an electron gas on a tubule," Physical Review B, vol. 48, no. 3, pp. 1947-1950, 1993.

[13] M. F. Lin and K. W. K. Shung, "Magnetoplasmons and persistent currents in cylindrical tubules," Physical Review B, vol. 48, no. 8, pp. 5567-5571, 1993.

[14] P. Longe and S. M. Bose, "Collective excitations in metallic graphene tubules," Physical Review B, vol. 48, no. 24, pp. 18239-18243, 1993.

[15] M. S. Kushwaha and B. Djafari-Rouhani, "Green-function theory of confined plasmons in coaxial cylindrical geometries: zero magnetic field," Physical Review B, vol. 67, no. 24, Article ID 245320, pp. 1-19, 2003.

[16] M. S. Kushwaha and B. Djafari-Rouhani, "Green-function theory of confined plasmons in coaxial cylindrical geometries: finite magnetic field," Physical Review B, vol. 71, no. 19, Article ID 195317, pp. 1-25, 2005.

[17] A. Moradi and H. Khosravi, "Plasmon dispersion in metallic carbon nanotubes in the presence of low-frequency electromagnetic radiation," Physics Letters A, vol. 371, pp. 1-6, 2007.

[18] A. Moradi and H. Khosravi, "Collective excitations in singlewalled carbon nanotubes," Physical Review B, vol. 76, no. 11, Article ID 113411, 4 pages, 2007. 
[19] A. A. Abrikosov, L. P. Gor'kov, and I. E. Dzyaloshinsky, Methods of Quantum Field Theory in Statistical Physics, PrenticeHall, Englewood Cliffs, NY, USA, 1963.

[20] G. D. Mahan, Many-Particle Physics, Plenum Press, New York, NY, USA, 1981.

[21] T. Ando, A. B. Fowler, and F. Stern, "Electronic properties of two-dimensional systems," Reviews of Modern Physics, vol. 54, no. 2, pp. 437-672, 1982.

[22] A. P. Prudnikov, Y. A. Brichkov, and O. I. Marichev, Integrals and Series, Nauka, Moscow, Russia, 1981. 

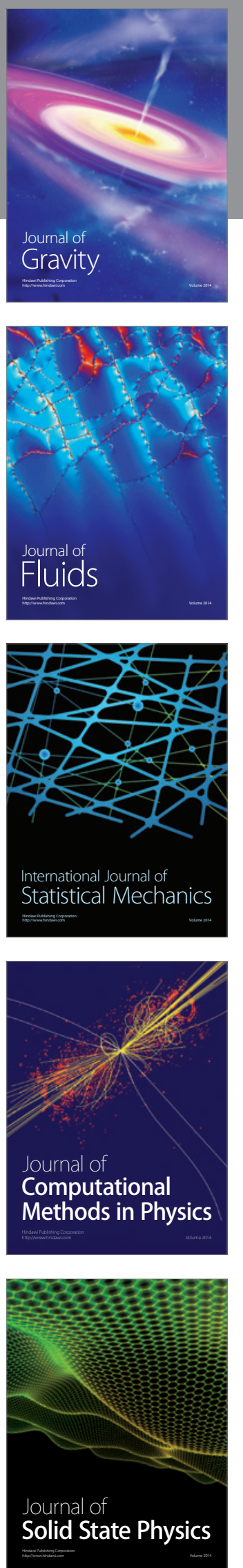

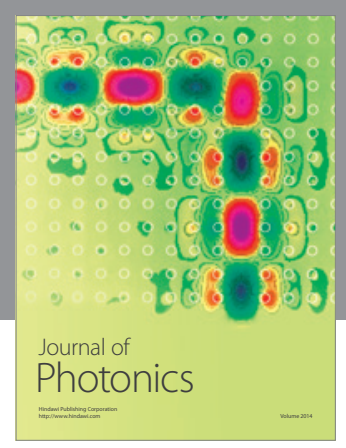

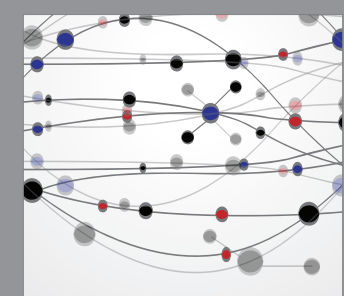

The Scientific World Journal
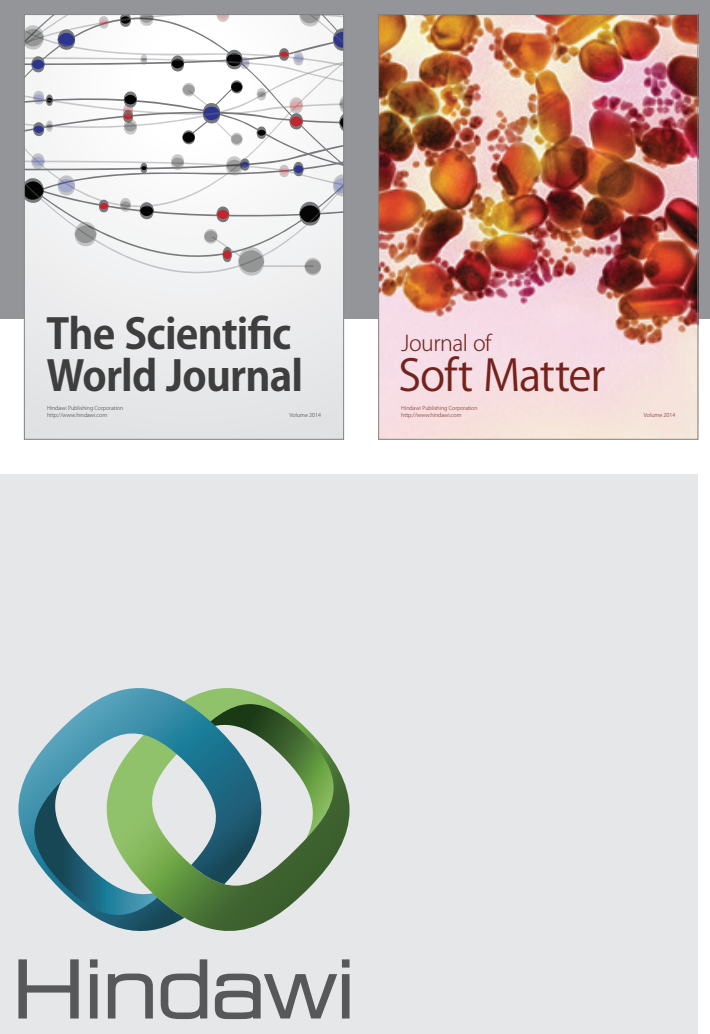

Submit your manuscripts at

http://www.hindawi.com
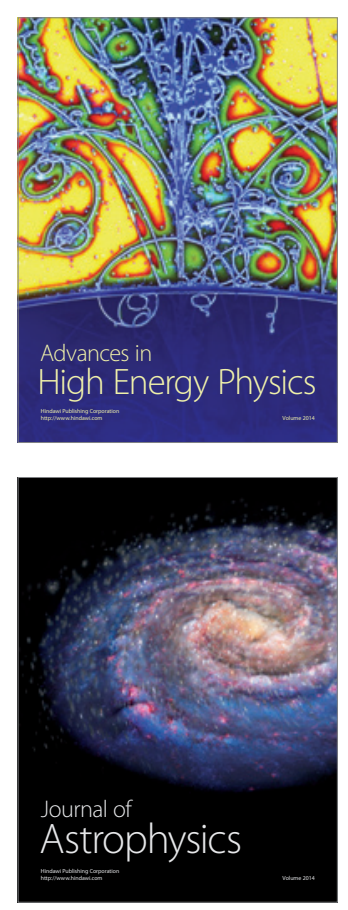
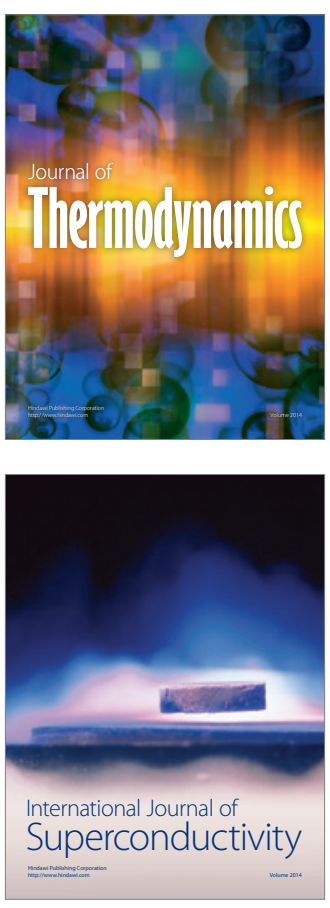
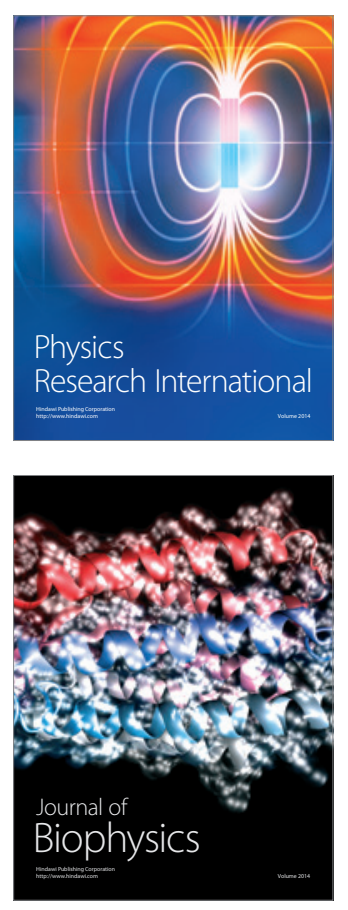
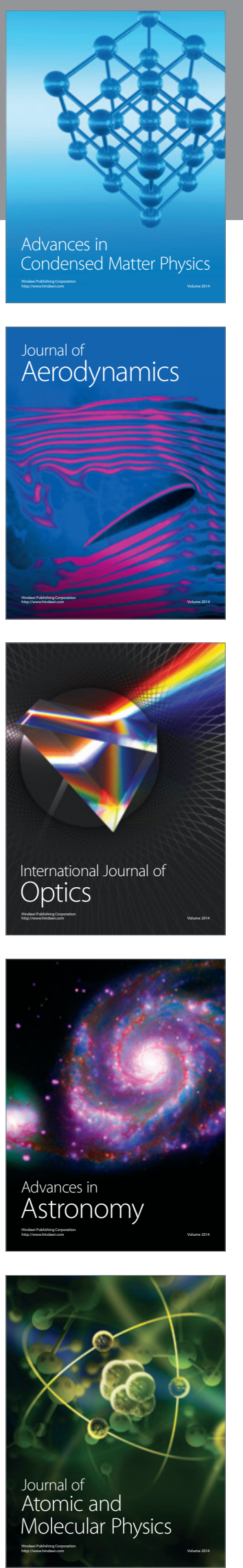